\section{Evidence for the Top Quark at Fermilab}

G. Bellettini and A.G. Clark summarise how the elusive top quark is being tracked down in spite of its production cross-section being two orders of magnitude less than that of the major background process.

\begin{tabular}{|lccc|}
\hline \multicolumn{4}{c}{ Summary of top-quark events at CDF } \\
& Dilepton & SVX & SLT \\
Number of events & 2 & 6 & 7 \\
Background expectations & $0.56_{-.13}^{+.25}$ & $2.3 \pm 0.3$ & $3.1 \pm 0.3$ \\
Probability that events come from background & $12 \%$ & $3.2 \%$ & $3.8 \%$ \\
\hline
\end{tabular}

On April 26th, the CDF experiment at the Fermilab $\bar{p} p$ Collider announced direct evidence for the production of top quarks in $\bar{p} p$ collisions [1]. The announcement noted the limited statistical significance of the result, due to the heavy mass of the top quark and its low production cross-section. If confirmed by further data now being collected, the result will provide a major confirmation of the Standard Model.

CDF previously placed a limit $m_{\mathrm{t}}>91$ $\mathrm{GeV} / \mathrm{c}^{2}(95 \% \mathrm{CL})$ on the top quark mass [2], and the combined indirect evidence from LEP experiments at CERN assuming the validity of the Standard Model gives $m_{\mathrm{t}}=164$ $\pm_{16}^{17}$ (stat) $\pm_{20}^{18}$ (sys) GeV/c $c^{2}$ [3]. Recently, the D0 experiment at the Fermilab Collider published an upper limit of $m_{\mathrm{t}}>131 \mathrm{GeV}(95 \%$ $\mathrm{CL}$ ) [4]. Fermilab's $\bar{p} p$ collider is the only operating accelerator having sufficient collision energy to produce the top, and the CDF and DO experiments are now collecting further data (CDF has operated since 1987, and D0 since 1992).

The CDF analysis used a data sample of integrated luminosity of 19.2 inverse picobarns $\left(\mathrm{pb}^{-1}\right)$ at a centre-of-mass collision energy of $1.8 \mathrm{TeV}$. In underlining the difficulty of the analysis leading to the evidence for top-quark production, the production crosssection for pairs quoted by CDF is extremely small $\left(13.6_{-4.8}^{+6.1} \mathrm{pb}\right)$, being more than two orders of magnitude less than the major background process, namely direct $\mathrm{W}^{ \pm}$production with jet activity.

\section{Two Approaches}

In $\bar{p} p$ collisions, the top quark is expected to be produced in pairs mainly by gluon-gluon fusion or $\bar{q} q$ annihilation (the latter being dominant for mass $\left.m_{\mathrm{t}}>100 \mathrm{GeV} / c^{2}\right)$. Recent estimates of the cross-section [5] vary typically between $38.9 \mathrm{pb}\left(m_{\mathrm{t}}=120 \mathrm{GeV} / c^{2}\right)$ and $4.2 \mathrm{pb}\left(m_{\mathrm{t}}=180 \mathrm{GeV} / c^{2}\right)$. For $m_{\mathrm{t}}>m_{\mathrm{W}}+m_{\mathrm{b}}$, the dominant decay is into a real $W$ and a b-quark (hence the W-pair plus b-quark pair configuration). Each $W$ subsequently decays to a lepton-neutrino pair, or a $\bar{q} q$ pair (see figure); each b-quark hadronises in a jet. The most abundant $(\approx 50 \%)$ final state has 6 hadron jets, but the background in this channel from known QCD processes is difficult to reject. The CDF analysis therefore used final states containing:

- Two oppositely charged, high transverse momentum $\left(p_{\mathrm{T}}\right)$ leptons (e or $\mu$ ), with asso-

Giorgio Bellettini works at the INFN, Pisa, and is Professor of Physics at Pisa University. He is the spokesperson of the Italian group (Bologna, Frascati, Padua, Pisa) participating in the CDF experiment.

Allan Clark is a member of the CDF Collaboration and is Professor of Physics, University of Geneva.
Top-quark production by $\bar{q} q$ annihilation, with the subsequent top-quark decay chain.

ciated missing transverse energy $E_{T}$ from undetected neutrinos, and at least two b-jets. - A high- $p_{\mathrm{T}}$ lepton (e or $\mu$ ), missing $E_{\mathrm{T}}$, and at least 3 jets to include at least one b-jet and at least one jet from W-decay.

The first (dilepton) analysis is the cleanest signature for $\mathrm{ft}$ pair production, but has low rate (only $5 \%$ of $\mathrm{tt}$ pairs decay in this configuration). Following detailed quality and kinematic selections on the leptons and missing

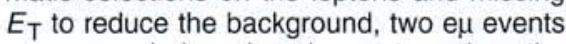
were recorded, and each event survives the subsequent requirement of at least two high$E_{\mathrm{T}}$ jets.

In the second analysis, the dominant backstandard QCD higher-order processes, with subsequent leptonic W-decay. The key to this analysis is an understanding of the background rate for $(\mathrm{W}+$ jets) production, and the identification of kinematic selections rejecting this background while retaining most of the $\vec{t} t$ signal. A total of 52 events with a high- $p_{T}$ lepton (>20 GeV/C) and at least a minimum of three jets (>15 GeV/c) were observed. As there is a large $W+$ jets background, analysis is needed to substantially enhance the $\overline{\mathrm{t}} \mathrm{t}$ signal. CDF analysis does this by attempting an event-by-event identification (tagging) of the b-quark jets by:

- Measuring the large (typically $\approx 130 \mu \mathrm{m}$ ) distance that high- $p_{\mathrm{T}}$ b-quarks travel before decay using a Si microvertex detector (SVX). A total of six events in the 52-event sample have at least one b-tag, while the background from known possible sources is estimated to be $2.3 \pm 0.3$ events.

- Searching with a second tagging algorithm (SLT) for a second low- $p_{\mathrm{T}}$ lepton resulting from b-quark decays. Of the 52 events, seven are tagged (including three events also tagged by the the first technique). The background from non- $\bar{t} t$ processes (including the dominant source of misidentified leptons) is estimated to be $3.1 \pm 0.3$ events.

\section{Several Significant Results}

The size and uncertainty, both statistica and systematic, of both types of detailed and difficult background estimates limit the claim

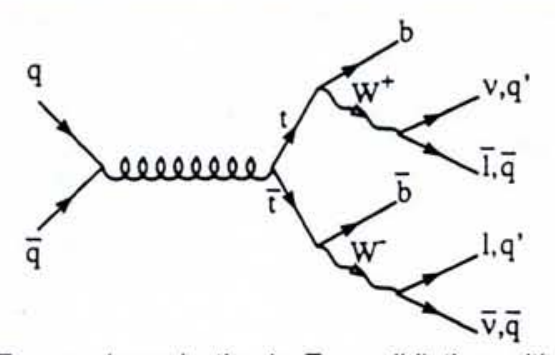
ground is the production of $\mathrm{W}+$ jets from of top-quark identification. The table summarises the event excess over expected background for each of the three analyses. The results of these analyses each show an excess of candidate events over the expected background, although the sizes of the separate excesses are not statistically significant. After accounting for tagging correlations, a combined probability of $0.26 \%$ (2.8 $\mathrm{\sigma}$ for a Gaussian probability) for the whole sample is quoted by CDF for the possibility that the events result from instrumental limitations or background.

If the excess over background is interpreted as resulting from $\bar{t} t$-production alone, then from the jet $E_{T}$, the missing $E_{T}$, and the lepton(s) $p_{\mathrm{T}}$, an evaluation has been made of the most likely mass for each event having at least four jets. Using various sets of these 4-jet events, a mass value of $m_{\mathrm{t}}=174+10$ (stat) ${ }_{-12}^{+13}$ (sys) $\mathrm{GeV} / \mathrm{c}^{2}$ is measured by CDF. This is the first time that a multi-body final state including jets of limited resolution in $E_{T}$ and missing $E_{T}$ has been successfully used for a mass evaluation.

The event kinematic properties have also been checked to be consistent with top production together with the estimated background. To exploit the different kinematic features expected for top-quark and background events, and in particular the jet energies and production angles, CDF made a seperate analysis looking for events having the features expected for top-quark events. An excess of these events over background expectations was found and it included a strong overlap with the sample having a tagged b-quark.

Several features of other rare events that do not easily fit a picture of $\bar{t} t$ production with QCD background were noted by CDF. In particular, the expected number of $W+4$-jet events for $E_{\mathrm{T}}$ only saturates the data (a $2 \sigma$ effect), and the number of tagged $(Z+$ jets) events exceeds expectations. Both observations are of limited statistical significance and more data are needed to resolve these questions.

[1] CDF Collaboration; submitted to Phys. Rev. D. [2] CDF Collaboration, Phys. Rev. Lett. 68 (1992) 447; Phys. Rev. D 45 (1992) 3921.

[3] CERN preprint CERN/PPE/93-157 (1993).

[4] D0 Collaboration; submitted to Phys. Rev. Lett.

[5] E. Laenen et al., Phys. Lett. 321B (1994) 254.

\section{Sn Identified}

Readers of last month's short article "Approaching ${ }^{100} \mathrm{Sn}$ in Decay Studies" will be interested to know that the GSI team reported shortly after we went to press the first observation of the doubly-magic nucleus ${ }^{100} \mathrm{Sn}$ (one of two that can probably be produced using present-day means). Projectile fragments generated by nuclear fragmentation of ${ }^{124} \mathrm{Xe}$ using the Institute's heavy-ion synchrotron SIS were separated in-flight and identified by measuring event-by-event the magnetic rigidity, the time-of-fight and the energy deposition [Schneider R. et al., Z. Physik A; submitted 27 April]. Future work aims to deduce the half-life and the decay mode, but more elaborate experiments will have to wait for a proposed upgrading of the SIS beam intensity. 